Article

\title{
Using Czech TCT to Assess Safety Impact of Deceleration Lane at Thai U-turns
}

\author{
Inder P. Meel ${ }^{1, a, *}$, Dussadee Satirasetthave ${ }^{2, b}$, Kunnawee Kanitpong ${ }^{3, c}$, \\ and Pichai Taneerananon ${ }^{4, \mathrm{~d}}$ \\ 1 Department of Transport Planning and Traffic Engineering, Faculty of Civil Engineering, Bauhaus- \\ University Weimar, Marienstrasse 13C, 99423, Weimar, Germany \\ 2 Department of Civil Engineering, Faculty of Engineering, Naresuan University, Phitsanulok, Thailand \\ 3 School of Engineering and Technology, Asian Institute of Technology, Pathumthani, Thailand \\ 4 Department of Civil Engineering, Faculty of Engineering, Prince of Songkla University, Songkhla 90110, \\ Thailand \\ E-mail: aipmeel@gmail.com (Corresponding author), bdussadee_s@outlook.com, ckanitpon@ait.ac.th, \\ dbreathislife@yahoo.com
}

\begin{abstract}
Purpose of this study is to evaluate safety impact of the deceleration lane at the Upstream Zone of at-grade U-turns on 4-lane divided Thai highways. A substantial speed reduction is required by vehicles for diverging and making $\mathrm{U}$-turn, and the deceleration lanes are provided for this purpose. These lanes are also providing a storage space for the U-turning vehicles to avoid unnecessary blockage of through lanes and reduce the potential of rear-end collisions. The safety at the U-turn is greatly influenced by the proper or improper use of the deceleration lanes. Subject to their length, full or partial speed adjustment can occur within the deceleration lane also the road users' behavior is influenced. To assess the safety impact, the four groups of U-turns with the varying length of deceleration lanes were identified.

Owing to limitation of availability and reliability of road crash data in Thailand, widely accepted Traffic Conflict Technique (TCT) was used as an alternative and proactive methodology. The U-turns' geometric data, traffic conflicts and volume data were recorded in the field at 8 locations, 8 hours per location.

Severity Conflict Rate (SCR) was assessed by applying a weighing factor (based on the severity grades according to the Czech TCT) to the observed conflicts related to the conflicting traffic volumes. A comparative higher value of SCR represents a lower level of safety. According to the results, increase in the functional length of the deceleration lane yields a lower value of SCR and a higher level of the road safety.
\end{abstract}

Keywords: Road safety, deceleration lanes, U-turn, severity conflict rates, CZECH traffic conflict technique.

ENGINEERING JOURNAL Volume 20 Issue 1

Received 1 March 2015

Accepted 6 July 2015

Published 29 January 2016

Online at http://www.engj.org/

DOI:10.4186/ej.2016.20.1.121 


\section{Introduction}

\subsection{Road Traffic Crash Trends in Thailand}

Road traffic crashes in developing as well as emerging countries tend to be one of the major reasons for fatalities and disabilities. Road traffic injuries take an enormous toll on individuals, communities as well as national economies. Middle-income countries, which are motorising rapidly, are the hardest hit. The economic growth in Thailand has resulted into an expanding network of roads and an increased number of the driving people. The growing number of vehicles on the roads, in turn, has contributed to significant increase in road crashes annually. In Thailand, the road traffic crash problem is regarded as one of the most serious social problems. The reported road traffic fatalities (in 2010) were 13766 and estimated GDP loss due to road traffic crashes was about 3\% [1]. Although there is a declining trend of traffic crashes in Thailand [2], yet the number of crashes are high among Southeast Asian countries [1]. Figure 1 shows traffic crash trend in Thailand.

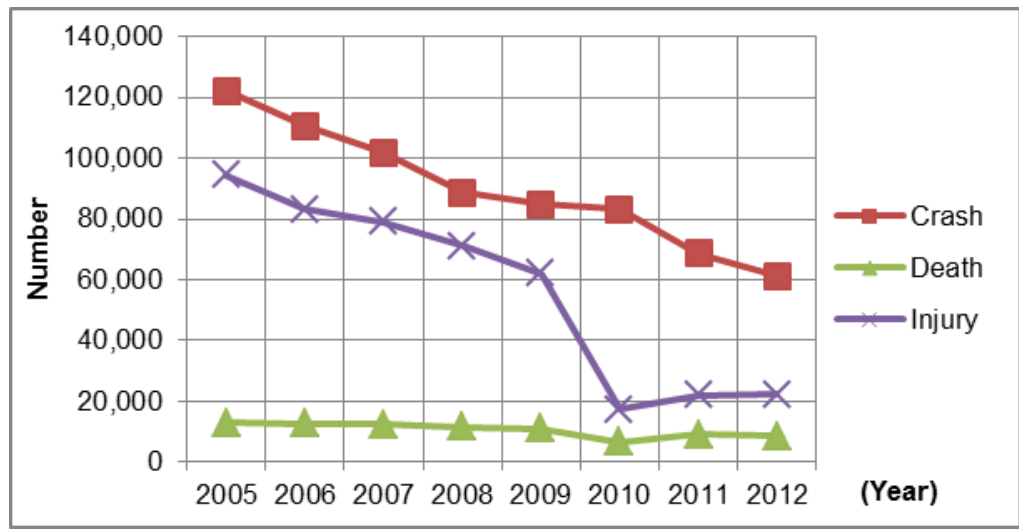

Fig. 1. Road traffic crashes trend in Thailand (Source: Prapongsena (2012)).

\subsection{Function of U-turn on the Thai Highways}

The median at-grade U-turns on the divided Thai Highways are provided for the U-turning movements to facilitate road users to join the opposite direction traffic stream. The basic functions of the median at-grade U-turns are shown in Fig. 2. The U-turns are also constructed to reduce the number of at-grade $\mathrm{T}$ and $\mathrm{X}$ junctions (to avoid direct right turn from highway to minor road and from minor road to highway (for left hand traffic)). The other purposes include the reduction in travel time for the emergency services, efficient law enforcement and for highway maintenance.

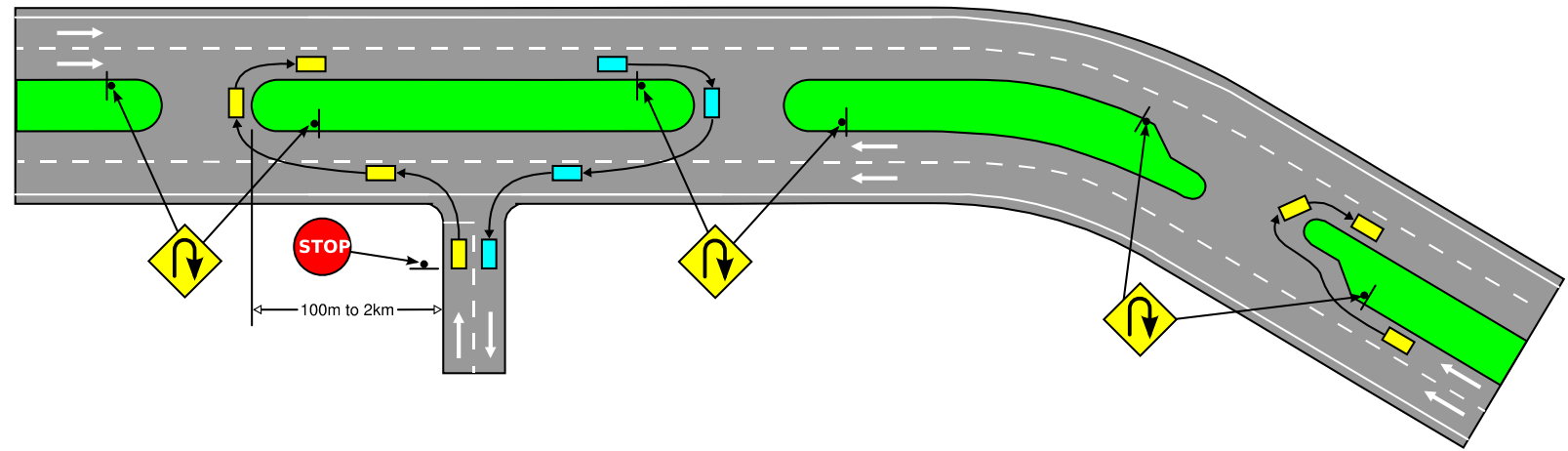

Fig. 2. The basic functions of the at-grade median U-turns.

\subsection{Road Safety at the U-turns}

The midblock U-turn junctions interrupt the through traffic movement. A substantial speed reduction is typically required by vehicles for diverging and making U-turns. The deceleration lanes are provided for this purpose. Safety at the U-turn is greatly influenced by the proper or improper use of deceleration lanes. 
Subject to their length, full or partial speed adjustment can occur within the deceleration lane, also road users' behaviour is influenced.

The deceleration lanes also provide a storage space for the U-turning vehicles to avoid unnecessary blockage of through lanes and reduce the potential of rear-end collisions. After arriving at the midblock median opening, the U-turning vehicle waits for a large enough gap to complete the U-turn maneuver. As the traffic volume increases on the through streams, the U-turning traffic confronts trouble in finding a sufficient gap to enter the other side of the driveway and a queue is formed at the deceleration lane which affects the through traffic movement in the same direction and, as a result, the drivers will experience longer travel and delay time. Sometimes the deceleration lane may get occupied completely, and may prompt an unsafe circumstance, where the vehicles will extend back to the highway (spillback), impeding the through movement traffic (see Fig. 3). Sometimes, it causes even worse circumstance where a parallel queue is formed at the inner through lane by the U-turning vehicles.

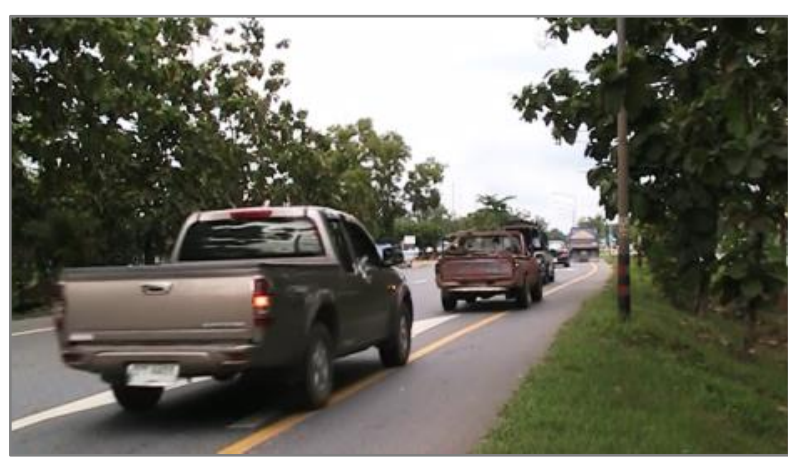

Fig. 3. Spillback effect by the queue of U-turing vehicles.

\subsubsection{Safety at Thai U-turns}

The U-turns are considered among the most road traffic crash prone sections of the Thai Highways, as illustrated in Figure 4.

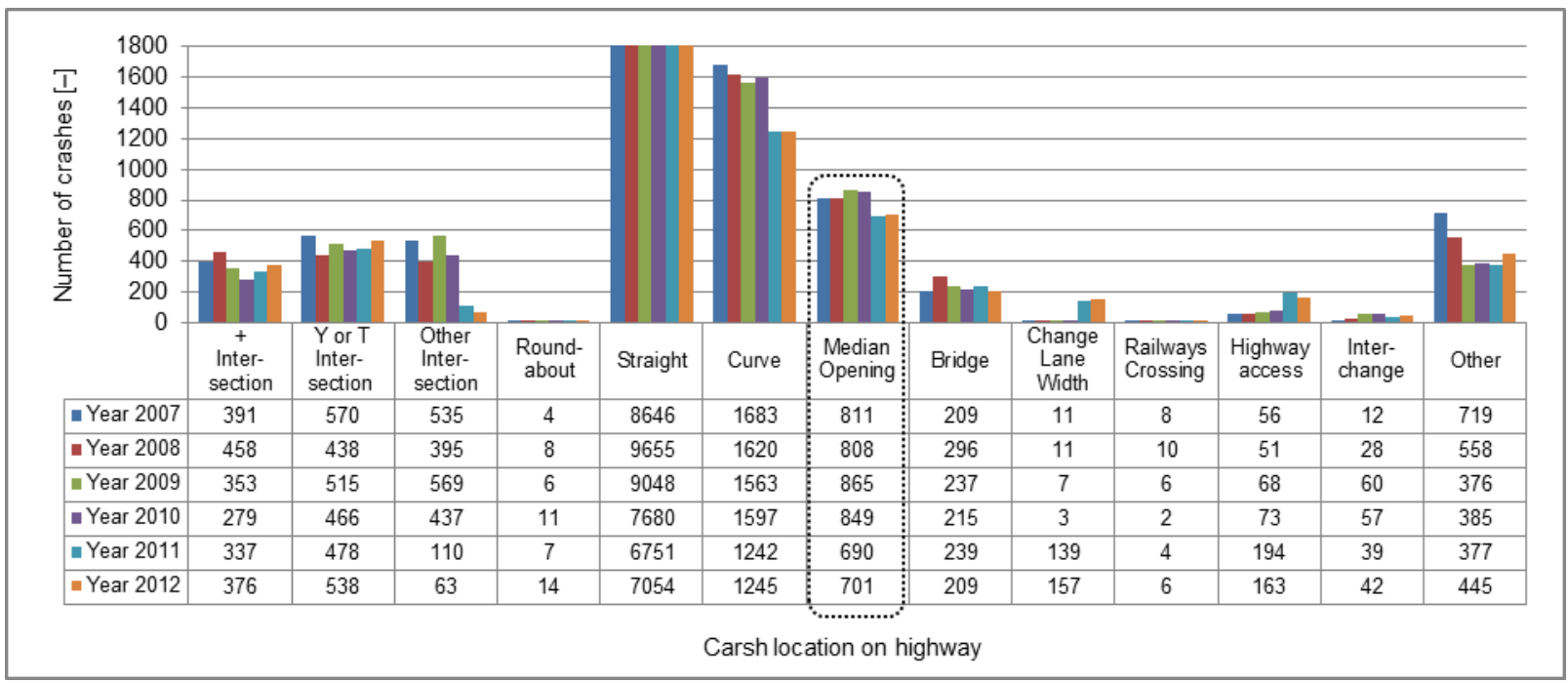

Fig. 4. Crash frequency by location on the Thai highways.

The numerous type of layout design of the U-turn are followed in Thailand. Some are standard (as per design guidelines of the Department of Highway) and the remaining are non-standard (based on the local design practice). Charupa (2011; [3]) stated that the U-turns are located near the entrance and exit of the local village and towns. Often, the various types of the U-turns confound unfamiliar drivers. In many areas, the U-turns are closely situated due to the requirement of service to the local residence. However, in some areas, the U-turns are located far from each other, causing illicit driving such as driving in the wrong direction to the closest U-turn point. 


\subsection{U-turn Design Consistency and Road Users' Behaviour}

The U-turn layout design varies with the application and dimensions of its components, such as auxiliary lanes (acceleration, deceleration and loons). At the U-turns, the acceleration (merging) lane and deceleration (diverging) lane are provided along inner lanes of the divided highways. Practically, the inner lanes are used for overtaking or dedicated to the vehicles moving with a higher speed. So, the merging and diverging maneuvers at the inner lanes make the U-turns susceptible towards traffic crash hazards. The application of variable length of deceleration lane produces inconsistent design characteristics of the road infrastructure. The shorter length of these lanes does not have enough space to make a comfortable lane change and decelerate, which may result in safety problem at the weaving and storage sections.

The posted speed limit at the Thai U-turns is same as mid-block speed limit (80 Kilometers per hour). The higher speed increases the severity of the impact of a collision. The conjunction of the high speed and the varying geometric conditions are major factors in crash causation in a high lethal crash rate.

The maneuvers of the motorcyclists for the U-turning movement make the study more complex and challenging. The Thai motorcyclists basically travel on the outer paved shoulder and they seldom use the inner auxiliary lanes for the U-turning movements. So they have to cross all through lanes in both the directions. Figure 5 is showing the typical driving maneuvers of the motorcyclist. The arrow with diamond head-marker (green) is pointing a motorcyclist, who is properly using the deceleration lane whereas the triangle head-marker (red) is pointing a user, who is not using the deceleration lane in a proper way.
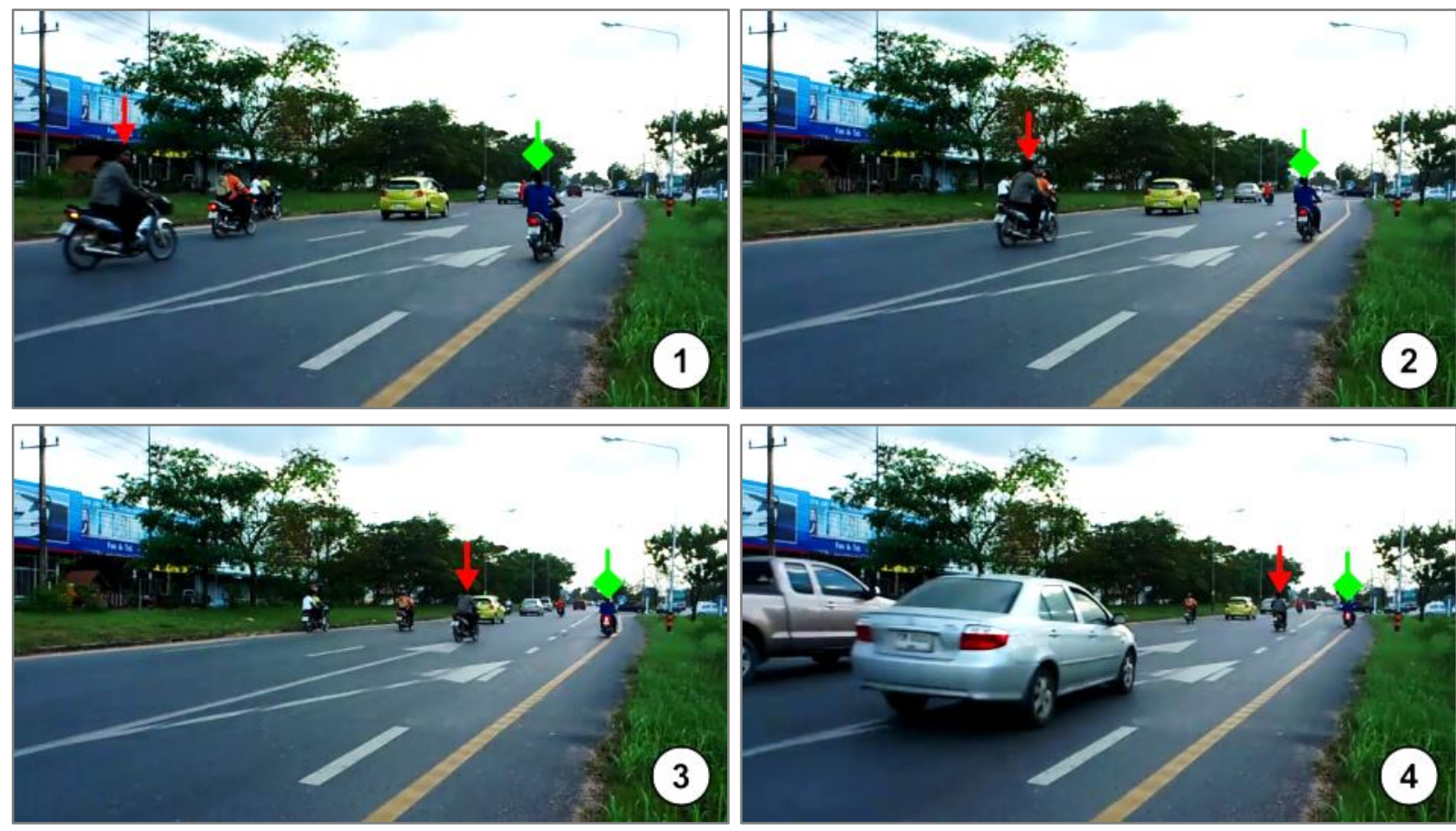

Fig. 5. Typical driving maneuvers by the Thai motorcyclists at a U-turn.

Similarly, the heavy commercial vehicles also, most of the times, travel on outer lanes. Therefore, before entering a U-turn facility, these vehicles have to cross the inner lane(s) and if the length of deceleration lane is not adequate, these face difficulty during weaving maneuvers (see Fig. 6). 

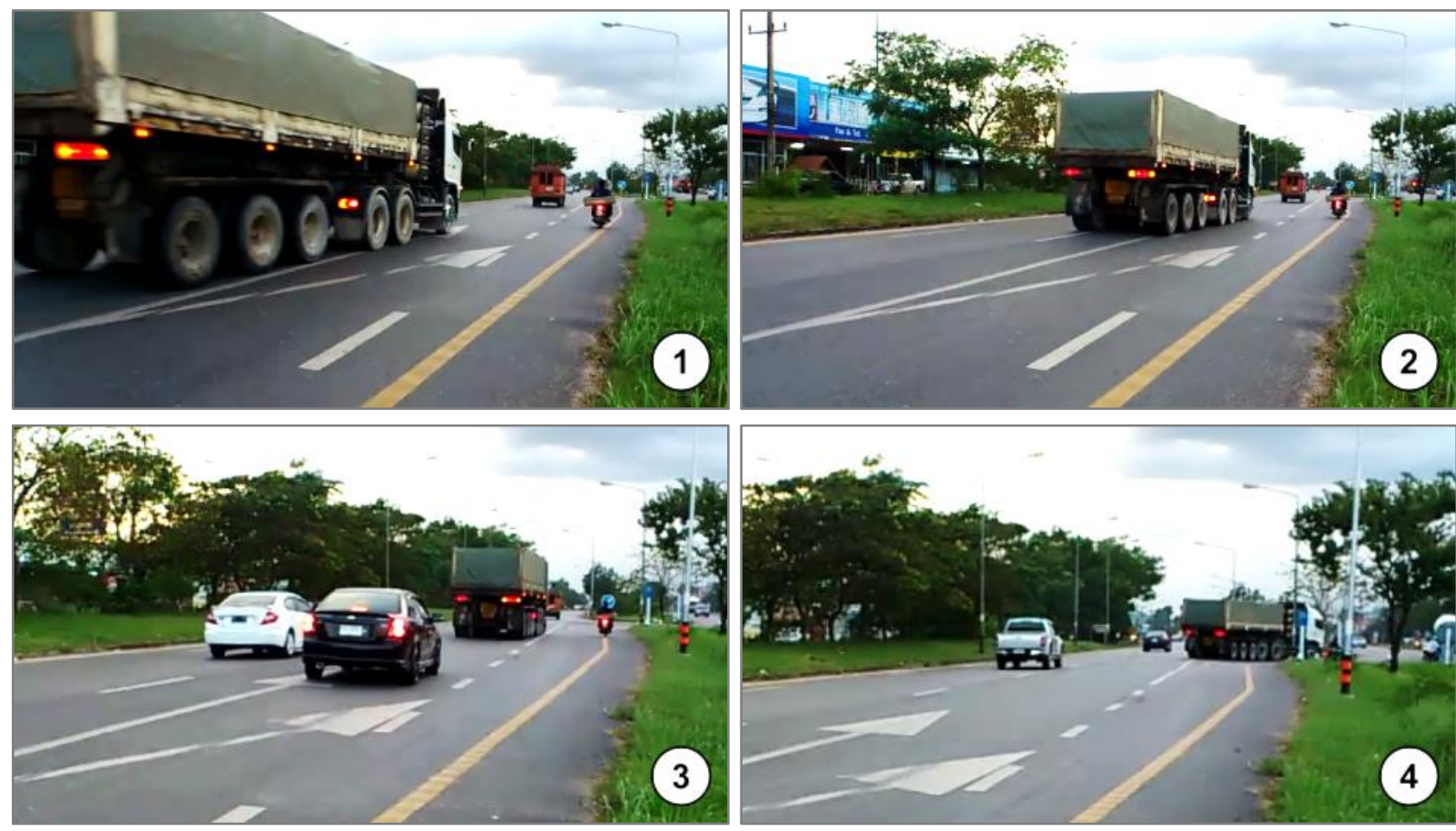

Fig. 6. Illicit driving maneuver by a heavy commercial vehicle while diverging at a U-turn

\section{Literature Review}

\subsection{Deceleration Lane Length Evaluation}

Romero (2010, [4]) studied 10 deceleration lanes with diverse lengths on freeways with no speed limit posted on the freeway. Therefore, different operating speeds were used $(130 \mathrm{~km} / \mathrm{h}, 120 \mathrm{~km} / \mathrm{h}, 100 \mathrm{~km} / \mathrm{h}$ and $80 \mathrm{~km} / \mathrm{h}$ ) to determine different configuration of lane lengths. A Potential Time to Lateral or Rear-End Collision (PTLRC) conflict indicator was developed using Traffic Conflict Technique. The study concluded that the shorter and the longer lane lengths are less safe. The intermediate lengths are the safest.

\subsection{Road Safety Measurement and Traffic Events}

The road safety refers to methods and measures adopted for reducing the risk of a person and vehicle, using the road network, being injured, death or harm and/ or damage to the material. Various tools and methods have been evolved for road safety assessment such as road safety audit, road safety inspection, crash modelling, conflict studies, monitoring road user behaviour, crash investigations and crash costing etc.

An event in traffic system is a term referring to all crashes, near-crashes, and incidents. The 'event' begins at the onset of the precipitating factor and ends after the evasive maneuver. Event Severity is a classification of the level of harm or damage resulting from an event and the five levels include a crash, near-crash, crash-relevant, proximity and non-conflict. For an event, road safety assessment crash and nearcrash events are mostly considered. The operational definitions of crash and near-crash are presented in the Table 1.

Table 1. Operational Definitions for the Event Severity Levels (Source: Feng Guo, [5]).

\begin{tabular}{ll}
\hline Severity Level & \multicolumn{1}{c}{ Operational Definition } \\
\hline Crash & $\begin{array}{l}\text { Any contact with an object, either moving or fixed, at any speed in which kinetic energy } \\
\text { is measurably transferred or dissipated. It includes other vehicles, roadside barriers, and } \\
\text { objects on or off of the roadway, pedestrians, cyclists, and animals. }\end{array}$ \\
Near-crash & $\begin{array}{l}\text { Any circumstance that requires a rapid, evasive maneuver by the participant vehicle or } \\
\text { any other vehicle, pedestrian, cyclist, and animal, to avoid a crash. A rapid, evasive } \\
\text { maneuver is defined as steering, braking, accelerating, and any combination of control } \\
\text { inputs that approaches the limits of the vehicle capabilities. }\end{array}$ \\
\hline
\end{tabular}




\subsection{Crashes Based Safety Analysis and its Limitations}

The road safety is commonly measured in terms of the number of traffic crashes and the consequences of these crashes with regards to their outcome in terms of severity. Traditionally, the level of safety of a specific location is measured by its history of rate of consequences (fatal, injury and property damage only) of crashes and traffic exposure. A most common challenge with this approach concerns the good quality and availability of crash data and the time-period required to statistically validate the success of different safety enhancing measures for the random and sparse nature of traffic crashes. As collisions are rare events, even at collision-prone locations, extended observation periods are required to determine stable trends. Also, not all crashes are reported, and the reporting level can vary from region to region. The quality and reliability of the crash data are important factors for providing the accurate results.

\subsubsection{Traffic Crash Data Management in Thailand}

In Thailand, the underreporting of crash data is widely acknowledged [6]. The principal agencies/ organisations such as the Royal Thai Police, the Department of Highways, and the Ministry of Public Health collect crash data only for the purposes of their interest. But, the integration of databases cannot be found in order to share data among various concerned agencies. Srirat's finding shows that 59.3 percent of under-reporting crash data was found from DOH data when compared to the police crash data in Nakhon Ratchasima province. Kowtanapanich (2006, [7]) mentioned that the standardisation, consistency and integrity are very poor as Royal Thai Police crash data is always kept in the narrative reports; also the accessibility to this data is limited to other users; this leads to getting the uncompleted or wrong information.

\subsection{Near-crash Events as an Alternative Approach}

If there are shortcomings (limitations of the availability and reliability of crash and traffic data) in collision based safety measures, the road safety analysis can benefit greatly from the methods that use an observable and non-collision based interactions. In order to perform an alternative and comprehensive form of safety analysis and to assess and predict levels of road safety at the specific types of traffic facilities, the Traffic Conflict Technique (TCT) is faster, more informative, and more resource effective method that yields valid and reliable safety measures in the short-term.

\subsubsection{Traffic Conflict Technique (TCT)}

A formalized definition of a traffic conflict was adopted as "an observable situation in which two or more road users approach each other in space and time to such an extent that there is a risk of collision if their movements remain unchanged" [8], and the observation method is formalized in the term as Traffic Conflict Technique (TCT).

The conflict safety indicators are particularly useful where there is an emphasis on the assessment and comparison of safety enhancement measures at specific traffic facilities and, in some cases, the interactions between specific road-user categories. The methodologies used to collect conflict data also make the results sensitive to site-specific elements related to roadway design and the dynamic as well as complex relationships among different traffic variables such as traffic flows, speed and proportions of turning movements [9].

\subsubsection{Validity and Reliability of TCT}

The reliability and validity are two issues strongly associated to the usability of TCT. These issues concern the lack of a consistent definition, their validity as a measure of traffic safety, and the reliability of their associated measurement technique. A number of studies have tried to address these issues ([10-13]). Some empirical studies found clear relationships between traffic conflicts and crashes [14]. Despite the concerns about those issues, traffic conflict techniques have been used in various studies to evaluate safety.

For the subjective TCT, the field observers are a source of error while collecting conflict data, due to the subjective nature of decision whether a given driving event is a conflict or not. Each observer is 
required to judge whether or not a situation is a conflict, resulting in variation in the grading of traffic conflicts by different people. As a result, the human-collected data was not necessarily accurate, especially if multiple observers were used. Nonetheless, traffic conflicts have been shown to have some correlation with crash frequency, and the consensus is that higher rates of conflicts correlate with lower levels of safety [15].

\subsubsection{Traffic Conflict Indicators and Conflict Severity Measurement}

The conflict indicators are defined as measures of crash proximity, based on the temporal and/or spatial measures that reflect the 'closeness' of road-users (or their vehicles), in relation to the projected point of collision. The objective evidence of a traffic conflict by the NCHRP definition is the evasive action which is indicated by a brake-light or a lane change affected by the offended driver. The first definition of a conflict was mainly based on brake light indications. A variety of observation methods has been developed to measure the severity of traffic conflicts, including the observation of driver behaviour and recording the number of near misses or avoidance maneuvers. Broadly, these can be classified into subjective and objective methods. Subjective methods include considerable judgment by the conflict observer and conflict severity taking into account the level of deceleration (weighted deceleration, which included longitudinalbraking and lateral-swerving-deceleration). To eliminate the subjectivity from the traffic conflict analysis, objective measures are used. The objective measures for traffic conflicts, having higher validity, include a cardinal or ordinal time-proximity dimension in the severity scale.

Mainly three indicators are widely recognized and discussed to assess the severity of conflicting situation, such as Time to Accident / Speed (TA/Speed), Time To Collision (TTC) and Post Encroachment Time (PET).

\subsubsection{Time to Accident / Speed (TA/Speed)}

The conflict measure is determined at a point in time and space when evasive action is first taken by one of the conflicting road-users [16]. The TA/Speed value is based on the necessity of a collision course and evasive action. The Time to Accident value (TA value) is the time that remains to an accident from the moment that one of the road users starts an evasive action if they had continued with unchanged speeds and directions. The Conflicting Speed is the speed of the road user taking evasive action, for whom the TA value is estimated, just before the start of the evasive action. An event with a low TA and a high speed value indicates an event with high severity.

\subsubsection{Time to Collision (TTC)}

The TTC value is also based on the necessity of a collision course. The proximity is estimated during the approach. The TTC is a continuous function of time as long as there is a collision course; the time required for two road users to collide if no evasive action is taken. The TTC ${ }_{\min }$ is the lowest value of TTC in the

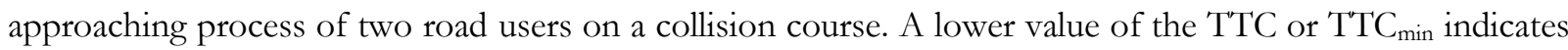
an event with high severity [17].

\subsubsection{Post Encroachment Time (PET)}

Post-encroachment time (PET) is the time between two vehicles on a near-collision course passing at a common point [18-19]. To measure PET, a collision course or an evasive action of road user(s) is not necessary. A lower PET indicates higher severity, and the minimum value is also the critical value.

\subsubsection{Grading Severity of Conflicts}

A conflict severity scale based on braking rates was proposed by Zimolong (1983, [20]), and four different conflict severity levels were specified: the first of these suggests a controlled use of brakes or controlled change of lanes to avoid collision; the second involves a severe use of brakes and/or an abrupt change of lanes; the third level involves emergency braking and fast driver reaction; whereas the fourth level involves collision.

Table 2 shows the characteristics of severity grades which are assigned to observed conflict situations based on severity of an evasive maneuvers. Situations of specific behaviour or misbehaviour have severity 
grade 0 , since they are situations of one user only and thus do not conform to a conflict definition. The Conflict Severity Grades 1, 2, 3 (highlighted in Table 2) are assigned to conflict according to the observed evasive maneuvers' severity, together with physical reactions and other characteristics. Obstruction and endangerment, used to distinguish between 2nd and 3rd severity grade, are defined according to the Czech TCT (2014, [21]).

Table 2. Characteristics of severity grades according to the Czech TCT (traffic conflicts are highlighted).

\begin{tabular}{llll}
\hline $\begin{array}{l}\text { Severity grade (CSG) } \\
\text { and description }\end{array}$ & Indicators & $\begin{array}{l}\text { Physical } \\
\text { reactions }\end{array}$ & Events (Related to vehicles) \\
\hline $0-$ (mis)behaviour & none & none & $\begin{array}{l}\text { breaking the rules without consequences, } \\
\text { misbehaviour of road users }\end{array}$ \\
\hline $1-$ slight conflict & low & common & fluent, controlled, predictable maneuvers \\
\hline $2-$ medium conflict & obstruction & sudden & pronounced, sudden, unpredictable maneuvers \\
\hline $3-$ severe conflict & endangerment & sharp & critical, emergency maneuvers \\
\hline
\end{tabular}

\subsubsection{Traffic exposure}

Salman and Al-Maita (1995, [22]) had research on three-legged intersections. The summation of all volumes entering the intersection and the square root of the product of the volumes that generated the conflicts were used to correlate conflicts and volumes. It was found that the correlation between the conflicts and the square root of the product of volumes was higher than that of the summation of volumes.

Yi and Thompson (2011, [23]) used a relationship between the traffic conflicts and the conflicting volumes at intersections as "the total number of traffic conflicts is proportional to the square root of the product of the conflicting volumes". This is referred to Sayed and Zein (1999, [24]), as the "product of entering vehicles" (PEV):

$$
P E V=\sqrt{\left(V_{2}\right) \times\left(V_{2}\right)}
$$

where: $V_{1}$ and $V_{2}$ represent the traffic volumes (vehicles/hour) of the two conflicting traffic streams.

\section{Methodology}

\subsection{Upstream Zone at a U-turn}

An Upstream Zone consists of through lanes and a deceleration lane as shown in Fig. 7. This zone is used by U-turning road users for diverging and deceleration from through traffic stream.

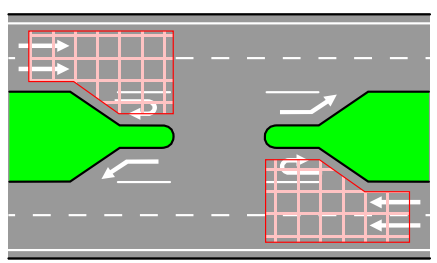

Fig. 7. Upstream Zones at a U-turn.

\subsection{Grouping of U-turns on the Basis of Length of Deceleration Lanes (DL)}

The four groups of U-turns on the basis of the length of the deceleration lanes were identified with the help of the field survey. Each U-turn has two deceleration lanes and each group has two U-turns. The functional length of DL $\left(L_{d f}\right)$ is defined as the sum of length of the section of the DL with full width $\left(L_{d c}\right)$ and half of the length of taper section $\left(L_{d t}\right)$ of the DL, as shown in Fig. 8 and presented in Table 3. 


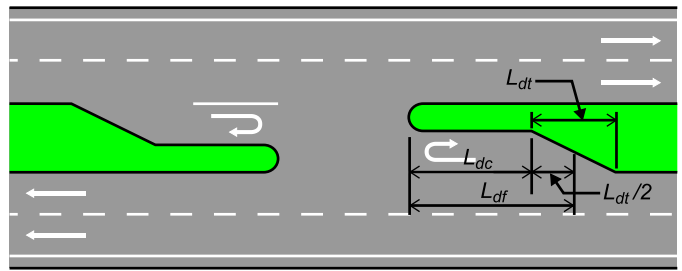

Fig. 8. The functional length of the deceleration lane.

Table 3. Grouping of the U-turns.

\begin{tabular}{ccccc}
\hline $\begin{array}{c}\text { U-turn } \\
\text { group }\end{array}$ & Length of DL & $\begin{array}{c}\text { Average length } \\
\text { of DL }\left(L_{d c}\right)\end{array}$ & $\begin{array}{c}\text { Average length of } \\
\text { tapper section }\left(L_{d t}\right)\end{array}$ & $\begin{array}{c}\text { Functional length of DL } \\
\left(L_{d f}=L_{d c}+L_{d t} / 2\right)\end{array}$ \\
\hline UT-1 & $0 \mathrm{~m}$ & $0.0 \mathrm{~m}$ & $0.0 \mathrm{~m}$ & $0.0 \mathrm{~m}$ \\
UT-2 & $22 \mathrm{~m}$ to $40 \mathrm{~m}$ & $32.5 \mathrm{~m}$ & $46.3 \mathrm{~m}$ & $55.2 \mathrm{~m}$ \\
UT-3 & $53 \mathrm{~m}$ to $70 \mathrm{~m}$ & $61.8 \mathrm{~m}$ & $88.5 \mathrm{~m}$ & $106.1 \mathrm{~m}$ \\
UT-4 & $90 \mathrm{~m}$ to $125 \mathrm{~m}$ & $105.5 \mathrm{~m}$ & $67.8 \mathrm{~m}$ & $139.4 \mathrm{~m}$ \\
\hline
\end{tabular}

\subsection{Conflicting Situations at Upstream Zone}

The traffic conflict situation means a situation where a driver brakes or swerves to avoid a collision. The conflicting situations are used to measure severity of conflict indicators as well as to identify type and category of conflict.

- Figure 9(a) shows a situation where a leading vehicle decelerates or stops for the U-turning maneuver and the following U-turning-vehicle has to decelerate or stop to avoid a collision. This situation causes a primary and diverging conflict and, the possible collision type could be rear-end.

- Figure 9(b) shows a situation where a leading vehicle decelerates or stops for U-turning maneuver and the following vehicle of through stream has to change lane with or without deceleration to avoid a collision. This situation causes a primary and diverging conflict, and the possible collision type could be rear-end.

- Figure 9(c) shows a situation where a leading vehicle moves from outer lane to the median opening for the U-turning maneuver and the following vehicle (through/ U-turning) has to decelerate or stop to avoid a collision. This situation causes a primary and crossing conflict, and the possible collision type could be side-swipe or angle.

- Figure 9(d) shows a situation where a leading vehicle decelerates or stops for U-turning maneuver and a following vehicle has to change lane with or without deceleration, and a third vehicle is also involved and it has to take an evasive action. This causes a secondary conflict situation and the possible collision type could be side-swipe or angle.

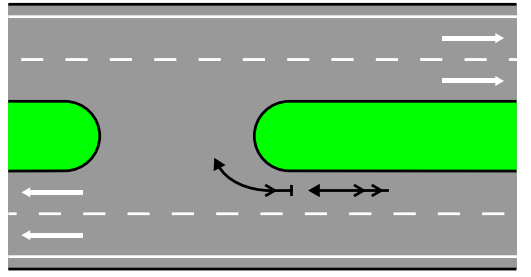

(a) Primary and diverging conflicting situation

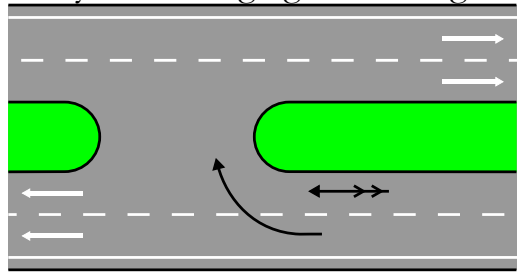

(c) Primary and crossing conflicting situation

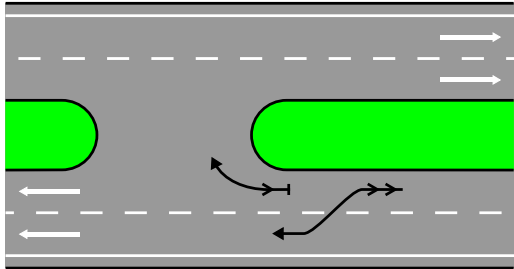

(b) Primary and diverging conflicting situation

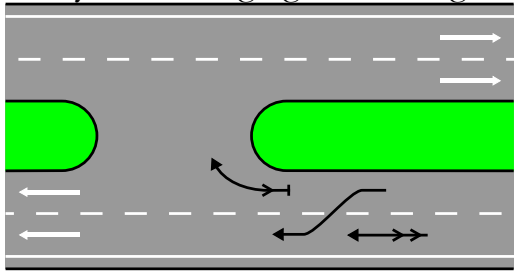

(d) Secondary conflicting situation

Fig. 9. Conflicting situations at the Upstream-zone. 


\subsection{Selection of Conflict Severity Indicators}

The U-turns have a distinct geometry, a longer conflict area in longitudinal direction and a higher operating speed. It makes difficult to judge the speed \& space between conflicting vehicles and to measure the severity of a conflict. This study focuses on the U-turns in non-built-up areas, where the elevated spots were unavailable to install a camera in a position to get the aerial view. Therefore, the use of the indicators TA/CS and TTC were not practically viable for this study. At the U-turns, the majority of conflicting events takes place due to the merging and diverging maneuvers, and the PET is suitable to measure the crossing conflict events. Therefore, the indicator PET was not used in this study.

Due to the above-mentioned constraints, a subjective approach was considered to measure the severity of traffic conflicts and the complexity of evasive action of the road users was considered as an indicator of conflict. For a comparative safety assessment, it is a justified approach to give weightiness (relative importance) to the conflict events to a higher level of seriousness. The purpose of using the weights is to put more emphasis on the severe conflicts than slight ones. The values of Weighting Coefficient of Conflict Severity Grade (CSG) were adopted from Czech TCT. The three levels of severity (seriousness) of traffic conflicts were adopted, as illustrated in Table 4, which were used to calculate the Severity Conflict Rate.

Table 4. Conflict Severity Indicators and Weighting Coefficients.

\begin{tabular}{llc}
\hline Severity & Indicators & Weighting Coefficient \\
\hline Slight & Sudden lane change or mere braking & 1 \\
Moderate & Intense deceleration of vehicle and almost stop & 2 \\
Severe & Hard braking or skid marks or braking sound & 3 \\
\hline
\end{tabular}

\subsection{Product of the Conflicting Volumes for the Upstream Zones $\left(\mathbf{P T T V}_{\mathrm{up}}\right)$}

The Product of Through and Turning (diverging) Volumes (PTTV $V_{u p}$ ) was computed for the U-turns as the traffic exposure to the observed conflicts for calculating the conflict rates. It is defined as "the square root of the product of (average hourly) traffic volumes of conflicting streams (through and diverging)".

\subsection{Conflict Numbers}

\subsubsection{Hourly Traffic Conflict Number (HCN)}

The Hourly Traffic Conflict Number (HCN) is defined as the number of observed conflicts at a zone divided by the number of observation hours for that zone. The three types of Hourly Traffic Conflict Numbers were computed based on the classification of severity of the conflicting situation as slight, moderate and severe.

\subsubsection{Average Hourly Traffic Conflict Number (AHN)}

The each U-turn has two Upstream Zones and two locations were investigated for the each U-turn type. Therefore, for the Upstream Zones of a group of U-turn type, the Average Hourly traffic conflict Number $(A H N)$ is defined as the summation of Hourly Traffic Conflict Numbers (HCN) at the Upstream Zones divided by the number of Upstream Zones in that group. Furthermore the Average Hourly traffic conflict Numbers were classified based on the severity of conflicting situations.

\subsection{Severity Conflict Rate $\left(\mathbf{S C R}_{\mathbf{u p}}\right)$ for the Upstream Zones}

The values of Weighting Coefficient (WC) for Conflict Severity Grade (CSG) from Table 4 were used as weighting coefficient for giving relative weightiness (importance) to the conflict events and to assess Severity Conflict Rates $(S C R)$. The $S C R$ is defined as a ratio of the summation of the product of the Average Hourly Slight, Moderate \& Sever Traffic Conflict Numbers $(A H N)$ and their respective value of the Weighting Coefficient for CSG to the Product of Through and Turning Volumes $\left(P T T V_{u p}\right)$ for the Upstream Zones. A higher value of $S C R$ at a traffic facility represents comparatively a lower level of traffic-safety. The $S C R$ for the Uturns was computed by the following equation: 


$$
S C R_{u p}=\frac{A H N_{u p-s l} \times W C_{s l}+A H N_{u p-m o} \times W C_{m o}+A H N_{u p-s e} \times W C_{s e}}{P T T V_{u p}}
$$

where:

$S C R_{\text {up }}$ - Severity Conflict Rate for a group of Upstream Zone,

$W C_{s l}-$ Weighting coefficient of $C S G$ for slight conflict $=1$,

$W C_{m o}-$ Weighting coefficient of $C S G$ for moderate conflict $=2$,

$W C_{s e}-$ Weighting coefficient of $C S G$ for severe conflict $=3$,

$A H N_{u p-s l}$ - Average Hourly Slight Traffic Conflict Numbers for Upstream Zones,

AHN $\mathrm{N}_{\text {up-mo }}$ - Average Hourly Moderate Traffic Conflict Numbers for Upstream Zones,

$A H N_{\text {up-se }}$ - Average Hourly Severe Traffic Conflict Numbers for Upstream Zones,

PTTV $V_{u p}$ - Product of through and turning (diverging) volumes for Upstream Zones.

\section{Data Type and Data Collection}

The gathered data relied on the form of the U-turn being studied and included traffic volumes, U-turning movement counts, using auxiliary lane counts, vehicle compositions, geometric data and traffic conflicts. From the classified four groups of U-turns, two locations for each group were selected and investigated throughout Thailand. Physical locations of selected U-turns are presented in Table 5 . The following basic requirements were applied while selecting the sites for the investigation:

- Located on the 4-lane divided highways,

- Outside of built-up area,

- Physically divided highways having median width between $0.5 \mathrm{~m}$ to $15 \mathrm{~m}$,

- Not to be located at the horizontal curve,

- Not to be located at the crest or sag curve,

- Not to be part of the T or X-junction,

- Not to be a grade separated design,

- No on-street parking,

- No pedestrian and bicycle traffic,

- No special design solution, and

- Posted or applicable speed limit 80 kilometer per hour.

Table 5. Physical locations of selected U-turns.

\begin{tabular}{crlrr}
\hline U-turn group & Site & Location & Latitude & \multicolumn{1}{c}{ Longitude } \\
\hline \multirow{2}{*}{ UT-1 } & A & Sadao, Songkhla & $6.647571^{\circ}$ & $100.427900^{\circ}$ \\
\cline { 2 - 5 } & B & Chang Wat, Ratchaburi & $13.601230^{\circ}$ & $99.833930^{\circ}$ \\
\hline \multirow{2}{*}{ UT-2 } & A & Hat Yai, Songkhla & $7.023115^{\circ}$ & $100.439300^{\circ}$ \\
\cline { 2 - 5 } & B & Hat Yai, Songkhla & $7.039420^{\circ}$ & $100.460800^{\circ}$ \\
\hline \multirow{2}{*}{ UT-3 } & A & Hat Yai, Songkhla & $7.054027^{\circ}$ & $100.479400^{\circ}$ \\
\cline { 2 - 5 } & B & Hat Yai, Songkhla & $7.066093^{\circ}$ & $100.489600^{\circ}$ \\
\hline \multirow{2}{*}{ UT-4 } & A & Chang Wat Chai Nat & $15.175760^{\circ}$ & $100.142200^{\circ}$ \\
\cline { 2 - 5 } & B & Phra Nakhon Si Ayutthaya & $14.155340^{\circ}$ & $100.291100^{\circ}$ \\
\hline
\end{tabular}

The traffic conflicts were recorded by the video cameras in the fields on working days during daylight hours ( 2 hours in the morning/ evening and 2 hours in the afternoon) avoiding adverse weather conditions. An aggregate of 16 hours video of the traffic operations data was recorded at each U-turn group. The recorded data was later reviewed in the laboratory for acquiring the traffic operations data.

\section{Results}

\subsection{Traffic Volumes}

At the upstream of a U-turn there are two types of traffic streams viz. through and diverging. 


\subsubsection{Hourly Traffic Volumes}

The observed hourly through and diverging traffic volumes are illustrated in Fig. 10 and the average hourly through and average hourly diverging traffic volumes are shown in Table 6.

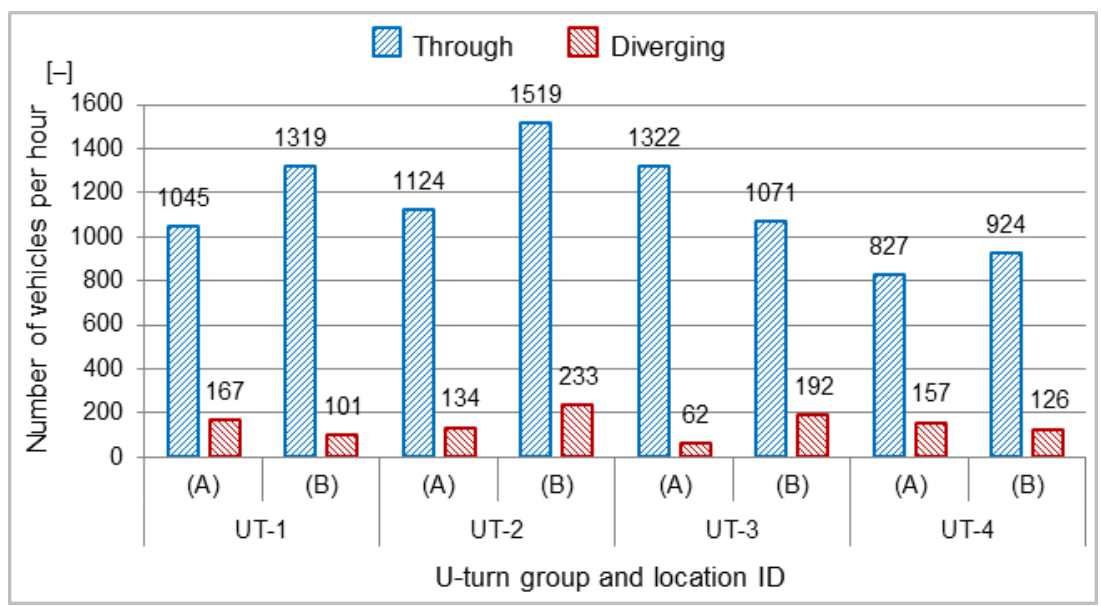

Fig. 10. The hourly traffic volumes at the upstream zone of the U-turn locations.

Table 6. The average hourly traffic volume at Upstream Zones and $\mathrm{PTTV}_{\text {up }}$.

\begin{tabular}{cccc}
\hline $\begin{array}{c}\text { U-turn } \\
\text { type }\end{array}$ & $\begin{array}{c}\text { Average hourly } \\
\text { through volume } \\
\text { [veh./hr] }\end{array}$ & $\begin{array}{c}\text { Average hourly } \\
\text { diverging volume } \\
\text { [veh./hr] }\end{array}$ & $\begin{array}{c}\text { Product of through } \\
\text { and diverging volumes } \\
\text { [veh./hr] }\end{array}$ \\
\hline UT-1 & 1182 & 134 & 398 \\
UT-2 & 1321 & 184 & 492 \\
UT-3 & 1197 & 127 & 390 \\
UT-4 & 875 & 141 & 352 \\
\hline
\end{tabular}

\subsection{Severity Conflict Rate $\left(\mathbf{S C R}_{\mathbf{u p}}\right)$}

The assessed Severity Conflict Rates for the Upstream Zones and the functional length of deceleration lanes $\left(L_{d f}\right)$ are illustrated in Table 7 and Fig. 11

Table 7. The Severity Conflict Indexes and functional length of the deceleration lanes $\left(\mathrm{L}_{\mathrm{df}}\right)$.

\begin{tabular}{|c|c|c|c|c|c|}
\hline $\begin{array}{l}\text { U-turn } \\
\text { group }\end{array}$ & $\begin{array}{c}\text { Avg. hourly } \\
\text { Slight } \\
\text { Conflicts nos. } \\
{[-]}\end{array}$ & $\begin{array}{c}\text { Avg. hourly } \\
\text { Moderate } \\
\text { Conflicts nos. } \\
{[-]}\end{array}$ & $\begin{array}{c}\text { Avg. hourly } \\
\text { severe } \\
\text { Conflicts nos. } \\
{[-]}\end{array}$ & $\begin{array}{c}\text { SCR } \\
(\times 100) \\
\\
{[\text { conflicts } / \text { veh] }}\end{array}$ & $\begin{array}{c}\text { Functional length } \\
\text { of deceleration lane } \\
\left(L_{d f}\right) \\
{[\mathrm{m}]}\end{array}$ \\
\hline UT-1 & 58.6 & 2.6 & 0.0 & 16.1 & 0.0 \\
\hline UT-2 & 32.8 & 1.5 & 0.0 & 7.3 & 55.2 \\
\hline UT-3 & 17.5 & 0.3 & 0.0 & 4.6 & 106.1 \\
\hline UT-4 & 10.6 & 0.1 & 0.0 & 3.1 & 139.4 \\
\hline
\end{tabular}

where $S C R_{\text {up }}$ - Severity Conflict Rate for the Upstream Zones. 


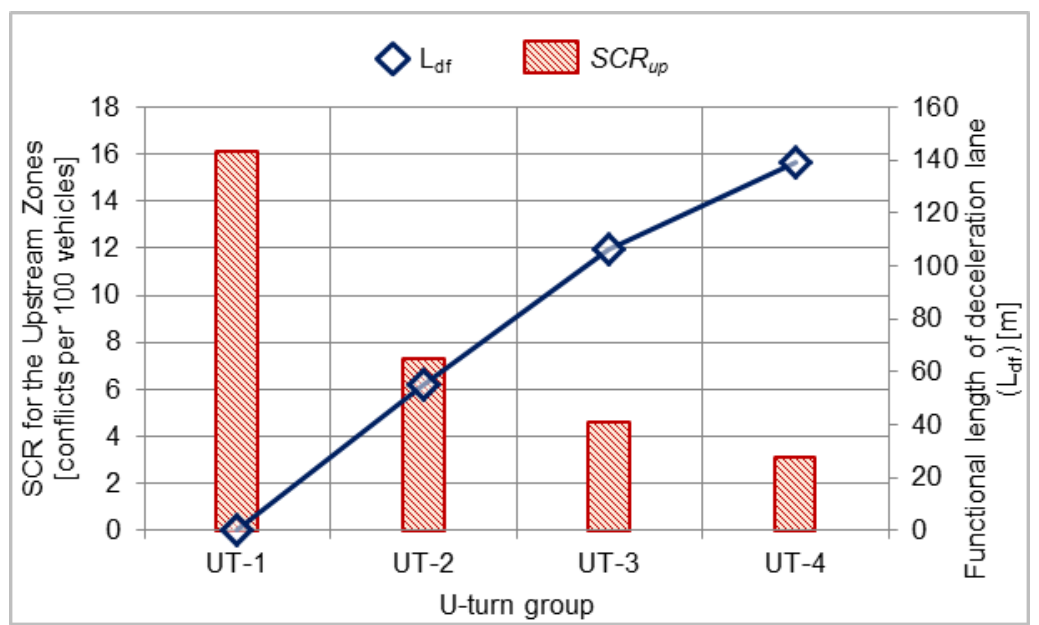

Fig. 11. The relationship between $\mathrm{SCR}_{\mathrm{up}}$ and $\mathrm{L}_{\mathrm{df}}$ (functional length of deceleration lanes).

\section{Conclusions}

A comparative higher value of the Severity Conflict Rate represents a lower level of safety at a traffic facility.

\subsection{Severity Conflict Rates for the Upstream Zones (SCR $\left.\mathbf{S p}_{\text {up }}\right)$}

The relationship between the functional length of the deceleration lane and the Severity Conflict Rates for the Upstream Zones $\left(S C R_{\text {up }}\right)$ could be comprehended from Fig. 11. It demonstrates that the increase in the functional length of the deceleration lane yields a lower value of $S C R_{u p}$ and a higher level of the road safety.

The U-turn group UT-1 has a zero functional length of the deceleration lanes; therefore it yields the highest value of $S C R_{u p}$ and a lowest level of road safety followed by the U-turn group UT-2 which has a 55 meters functional length of the deceleration lane. The U-turn group UTA has the longest functional length of the deceleration lane, $139 \mathrm{~m}$; therefore it demonstrates lowest value of $S C R_{u p}$ and yields the highest level of the road safety.

The Standard Drawing from the 'Department of Highways' of Thailand illustrates the length of deceleration lane as 160 meters (including 60 meters length of taper section), but, among identified U-turn types, only the UTA has these dimensions. The rest of U-turn types have a shorter length of the deceleration lanes. A shorter length of deceleration lane does not provide adequate space for comfortable deceleration and lane change for the diverging vehicles; this may result in a lower level of road safety for the weaving and storage maneuvers of diverging vehicles.

\section{Recommendations}

At the U-turn group UT-1, no deceleration lane has been applied and it yields a lower level of road safety. Therefore, these should be modified as early as possible and should not be applied to the future projects. The literature survey indicates that, for the deceleration lanes, the shorter and the longer lane lengths are less safe, and the intermediate lengths are safest. At a U-turn, the U-turning vehicles have to stop and wait before merging into opposite side traffic streams. Therefore, if there is a high volume of the U-turning vehicles, in that condition, the length of deceleration lanes must be adequate for the storage of these vehicles.

Furthermore, there are some areas of this research, which are needed to be enhanced in future studies. The several objective methods for measuring the level of conflict severity, such as Time-to-Collision, PostEncroachment-Time should be considered as an important factor for predicting the conflict severity and reducing dependency of human judgments. For the future assessment of the comparative road safety at the U-turns, in contrast to subjective Traffic Conflict Techniques, the use of micro-simulation software and programs could be the advanced technological tools to produce the results with a higher level of accuracy, reliability and validity. 
Also, there is a very serious need for the establishment of a well-structured and systematic traffic crash data system in Thailand for improving the road safety strategies to ensure timely \& quality results. This study has used a surrogate and subjective to human judgment approach, which is frequently debated by the experts and practitioners for its reliability and subjectivity.

\section{References}

[1] WHO Committee, "Global status report on road safety 2013: Supporting a decade of action,” World Health Organisation Staff, Eds., World Health Organization, 2013.

[2] P. Prapongsena, I. Sangphet, and S. Kraisingsom, "Traffic accident on national highways in 2012," Bureau of Highway Safety, Department of Highway, Thailand, Bangkok, Annual Report, 2012.

[3] T. Charupa, "Highway efficiency improvement: Thailand' s route no 4-Case study," 2011.

[4] M. A. Romero and A. García, "Deceleration lane length evaluation based on a new conflict indicator," in Proc. 4th International Symposium on Highway Geometric Design, Valencia, Spain, 2010.

[5] F. Guo, S. G. Klauer, M. T. McGill, and T. A. Dingus, "Evaluating the relationship between nearcrashes and crashes: Can near-crashes serve as a surrogate safety metric for crashes?," NHTSA, US Department of Transportation, vol. 811, p. 382, 2010.

[6] P. Srirat, "Underreporting of road casualty accident data: A case study of highways in Nakhon Ratchasima, Thailand," Ph.D. dissertation, School of Civil Engineering, Asian Institute of Technology, 2008.

[7] W. Kowtanapanich, "Development of the GIS-based traffic accident information system integrating police and medical data: A case study in Khon Kaen, Thailand," Ph.D. thesis, Asian Institute of Technology, 2006.

[8] F. Amundsen and C. Hyden, "The Swedish traffic conflict technique," in Proceedings of First Workshop on Traffic Conflicts, Oslo, 1977, pp. 1-5.

[9] J. Archer, "Indicators for traffic safety assessment and prediction and their application in microsimulation modelling: A study of urban and suburban intersections," Ph.D. thesis, Royal Institute of Technology, Stockholm, Sweden, 2005.

[10] M. J. Williams, "Validity of the traffic conflicts technique," Accident Analysis \& Prevention, vol. 13, pp. 133-145, 1981.

[11] E. Hauer, "Traffic conflicts and exposure," Accident Analysis \& Prevention, vol. 14, no. 5, pp. 359-364, 1982.

[12] D. J. Migletz, W. D. Glauz, and K. M. Bauer, "Relationships between traffic conflicts and accidents volume I-Executive Summary," Federal Highway Administration, 1985.

[13] E. Hauer and P. Garder, "Research into the validity of the traffic conflicts technique," Accident Analysis \& Prevention, vol. 18, no. 6, pp. 471-481, 1986.

[14] W. D. Glauz, K. M. Bauer, and D. J. Migletz, "Expected traffic conflict rates and their use in predicting accidents," Transportation Research Record: Journal of the Transportation Research Board, vol. 1026, pp. 1-12, 1985.

[15] D. Gettman, L. Pu, T. Sayed, and S. G. Shelby, "Surrogate safety assessment model and validation: Final report," Publication FHWA-HRT-08-051. FHWA, U.S. Department of Transportation, 2008.

[16] S. R. Perkins and I. H. Joseph, "Traffic conflict characteristics accident potential at intersections," Research Laboratories, General Motors Corporation, 1967.

[17] J. C. Hayward, "Near-miss determination through use of a scale of danger," Highway Research Record, vol. 384, 1972.

[18] B. L. Allen, B. T. Shin, and P. J. Cooper, "Analysis of traffic conflicts and collisions," Transportation Research Record, No. HS-025 846, 1978.

[19] R. Van der Horst and J. Kraay, "The Dutch Conflict Observation Technique--DOCTOR," in Proceedings of the Workshop "Traffic Conflicts and Other Intermediate Measures in Safety Evaluation," Budapest, Hungary, 1986.

[20] B. Zimolong, H. Erke, and H. Gstalter, Traffic Conflicts at Urban Junctions: Reliability and Validity Studies. Brunswick, Germany: University of Technology, 1983.

[21] J. Ambros, R. Turek, and J. Paukrt, "Road safety evaluation using traffic conflicts: Pilot comparison of micro-simulation and observation," in Proc. International Conference on Traffic and Transport Engineering, Belgrade, Nov. 27-28, 2014. 
[22] N. K. Salman and K. J. Al-Maita, "Safety evaluation at three-leg, unsignalized intersections by traffic conflict technique," Transportation Research Record, vol. 1485, pp. 177-185, 1995.

[23] Y. Yi and M. K. Thompson, "Quantifying the impact of coupling in axiomatic design: Calculating the coupling impact index for traffic intersections," in Proceedings of the 6th International Conference on Axiomatic Design, Daejeon, Korea, 2011.

[24] T. Sayed and S. Zein, "Traffic conflict standards for intersections," Transportation Planning and Technology, vol. 22, pp. 309-323, 1999. 